\title{
Hypercapnic acidosis microenvironment promoted epithelial-to-mesenchymal transition through activating ASIC3 in lung adenocarcinoma
}

\author{
Dingde Long \\ the First Affiliated Hospital of Nanchang University \\ Xingjun Fang \\ the First Affiliated Hospital of Nanchang University \\ Peihua Yuan \\ the First Affiliated Hospital of Nanchang University \\ Hongtao Li \\ Virginia Commonwealth University \\ Liangchao. Qu ( $\nabla$ liangchao.qu@outlook.com) \\ the First Affiliated Hospital of Nanchang University
}

\section{Research Article}

Keywords: Epithelial-mesenchymal transition, Hypercapnic acidosis, Microenvironment, Lung cancer, acid-sensing ion channels 3

Posted Date: February 8th, 2022

DOI: https://doi.org/10.21203/rs.3.rs-1320137/v1

License: (c) (1) This work is licensed under a Creative Commons Attribution 4.0 International License. Read Full License 


\section{Abstract}

Background Epithelial-mesenchymal transition (EMT) plays a major role in tumor invasion and metastasis, which is associated with poor survival in surgically resected lung cancer. Tumor microenvironment, especially extracellular acidity is a favorable factor for tumor progression and metastatic spread, and participates in EMT. However, the effects of Hypercapnic acidosis (HCA) microenvironment on EMT of lung cancer still unknown.

Materials and Methods The acid-sensing ion channels (ASICs) gene expression and prognostic value in lung adenocarcinoma (LUAD) and lung squamous cell carcinoma (LUSC) were evaluated using online database. Human lung adenocarcinoma A549 cells were cultured in 10\% CO2 and 90\% air for $48 \mathrm{~h}$ to assess the effects of the HCA microenvironment. Transwell assay and wound-healing assay of A549 cells were performed to determine the effects on EMT.

Results The mRNA expression levels of ASIC3 may be useful for prediction of lung cancer patient overall survival. The expression of ASIC3 significantly increased, and the invasive and migrate ability of A549 cells was promoted after exposing in HCA microenvironment (10\% CO2 and $90 \%$ air), and inhibited when ASIC3 was blocked.

Conclusion HCA microenvironment induced EMT in lung cancer cells through ASIC3, which may have influence on the FP and OS of lung cancer patients.

\section{Introduction}

Among the malignant tumors, the mortality rate of lung cancer ranks first in the world. The incidence and mortality of lung cancer in China have also continued to increase in the past few years [1,2]. According to the prediction of the World Health Organization (WHO), the number of deaths from lung cancer will exceed one million by 2025, and the number of patients will rank first in the world [3]. Lung cancer is one of the malignant tumors with the worst prognosis. When more than $80 \%$ of patients come to the hospital for treatment, they have lost the best time for surgery and multidisciplinary treatment [4]. Lung cancer is the highest number of cancer-related deaths in the world, which the most important reason is tumor cell metastasis[5, 6]. Metastasis is a multi-step process, which includes cancer cell epithelial-mesenchymal transition (EMT), separation from the primary tumor, migration to nearby tissues or distant organ tissues, and eventually tumor cells spread [7]. Once the cancer cells metastasize, treatment will become very difficult and the chance of survival decreases [8]. Therefore, EMT plays a major role in tumor invasion and metastasis, which is associated with poor survival in surgically resected lung cancer $[9,10]$. However, the exact mechanism of EMT in lung cancer has not been fully elucidated.

The tumor microenvironment is an extremely complex internal environment system, composed of tumor interstitial cells, cell metabolites, and various physical and chemical factors such as power of hydrogen $(\mathrm{pH})$, hypoxia, and interstitial fluid pressure [11].Recent studies have been showed that acidity is an inherent feature of the tumor microenvironment $[12,13]$. Extracellular acidity is a favorable factor for 
tumor progression and metastatic spread, and participates in EMT [14]. Hypercapnic acidosis (HCA) is a common clinical comorbidity of lung disease, and HCA has been proven to improve the prognosis of patients with acute lung injury/acute respiratory distress syndrome (ALI/ARDS) [15, 16]. Meanwhile, HCA has some harmful effects such as suppressing innate immunity/host defense $[17,18]$. HCA is a common complication of lung cancer patients, but it is not clear how HCA affects lung cancer. In this study, we worked on how HCA microenvironment affect EMT of human lung adenocarcinoma cells (A549 cells).

Acid-sensing ion channels (ASICs) is a kind of sense $\mathrm{H}+$ channel, which is an epithelial sodium channel /degenerin superfamily members and activated by the $\mathrm{pH}[19,20]$. This channels mainly transit? through $\mathrm{Na}+$, and is less permeable to $\mathrm{K}+$ and Ca2+. Six ASICs have been cloned so far: ASIC1a, ASIC1b, ASIC2a, ASIC2b, ASIC3 and ASIC4 [21]. It has been reported that acidic condition stimulates tumor cell invasion which may involve lysosomal proteases, extracellular matrix degradation and promotion of normal cell death [22]. Moreover, ASIC1 and ASIC3 promoted EMT of cancer cells during acidic microenvironment by transducing the acidic to elevation of intracellular $\mathrm{Ca} 2+$ concentration [23]. It has been showed that ASIC3 plays an important role in lipopolysaccharide-induced ALI in vivo study [24]. However, until now it has not been examined on the effects of ASICs on lung cancer. In this study, we took advandge of bioinformatics data analysis and found that the mRNA expression levels of ASIC3 might be useful for prediction of lung cancer patient overall survival. Furthermore we tested our hypothesis that exposure of lung cancer cells to HCA microenvironment induces an EMT, and the effects of ASIC3 in this process, thereby increasing the potential for metastasis of A549 lung cancer cells.

\section{Materials And Methods}

\section{GEPIA (Gene Expression Profiling Interactive Analysis) dataset}

To analysis the ASICs gene expression in lung cancer, GEPIA was used which is an interactive web server for analyzing the RNA sequencing expression data (http://gepia.cancer-pku.cn/) [25]. Tumor data of lung adenocarcinoma (LUAD) and lung squamous cell carcinoma (LUSC) were selected to analysis the expression on box plots and pathological stage plot, considering adjusted $p<0.01$ and log2FC $>1$ as a significant difference.

\section{The Kaplan-Meier plotter}

The prognostic value of ASICs mRNA expression in lung cancer patients was evaluated using KaplanMeier Plotter database (http://kmplot.com/analysis/index.php?p=service\&cancer=lung) [26]. 2437 patient samples were split into two groups by median expression (high vs. low expression) and assessed by a Kaplan-Meier survival plot. The overall survival (OS), progression-free survival (FP), and post progression survival (PPS) of patients with lung cancer were analyzed with the hazard ratio (HR), $95 \%$ confidence intervals $(\mathrm{Cl})$ and logrank $p$ value.

\section{Immunohistochemistry}


Immunohistochemical staining was done on the paraffin-embedded cancer tissue. Paraffin sections of 3$\mu \mathrm{m}$ thick were cut and probed by $0.3 \%$ hydrogen peroxide for $30 \mathrm{~min}$ and the sections were incubated with the primary antibodies ASIC1, ASIC2, ASIC3 andASIC4 which were purchased from Cell Signaling Technology (Danvers, MA, USA) respectively (1:100 to 1:150 in PBS, overnight at $\left.4^{\circ} \mathrm{C}\right)$. Then the sections were incubated with the biontinylated secondary antibody (1:200; Santa Cruz Biotechnology, Santa Cruz, CA) for $30 \mathrm{~min}$ at $37^{\circ} \mathrm{C}$ and covered by DAB and mounted with Vectashield mounting medium (Vector Laboratories). Subsequently, all fields were observed under light microscopy (Olympus, Tokyo, Japan).

\section{Cell culture and reagents}

Human lung adenocarcinoma A549 cells were purchased from ATCC Cell Bank and were cultured in Dulbecco's modified Eagle's medium (DMEM) with 10\% fetal bovine serum (FBS) at $37^{\circ} \mathrm{C}, 5 \% \mathrm{CO} 2$ and 95\% air. To assess the effects of the HCA microenvironment, the cells were cultured in 10\% CO2 and $90 \%$ air [27], the pH and the pressure of $\mathrm{CO} 2$ in the medium were $6.69 \pm 0.02$ and $71.01 \pm 3.17 \mathrm{mmHg}$ respectively. To block ASIC3, aminloride (Sigma) was added to medium at the final concentrations of 100 $\mu \mathrm{M}$ for $48 \mathrm{~h}$. The cells were seeded in 12-well tissue culture plates. The rabbit anti-human antibody ASIC3 was purchased from Cell Signaling Technology (Danvers, MA, USA), and anti-rabbit secondary antibodies was purchased from KangChen Bio-tech (Shanghai, China). BCA protein assay kit was purchased from Beyotime Biotechnology (Shanghai, China).

\section{Immunofluorescence analysis}

A549 cells were plated in 24-well plates and fixed with $0.1 \%$ Triton for $30 \mathrm{~min}$ at $4{ }^{\circ} \mathrm{C}$. After washed with PBS, A549 cells were treated with $5 \%$ bovine serum albumin for $60 \mathrm{~min}$ at room temperature and then stained with primary antibody of ASIC 3 at $4{ }^{\circ} \mathrm{C}$ overnight. The primary antibody was mouse anti-human vimentin (DAKO). The secondary antibody FITC (goat anti-mouse, AbCAM) was diluted $1: 200$ in PBS, and incubated for $60 \mathrm{~min}$ at $4{ }^{\circ} \mathrm{C}$, and then slides were mounted onto slides with $1 \%$ 4'6-diamidine-2'phenylindole dihydrochloride (DAPI) for $10 \mathrm{~min}$. Cell images were visualized using a scanning confocal fluorescence microscope (Olympus Co, Japan).

\section{Transwell assay and wound-healing assay}

A549 cells migration were assessed by using 24-well transwell chambers (Corning, Shanghai, China) with 8- $\mu \mathrm{m}$ pore size transwell filters (6.5-mm diameter; Corning Inc., Corning, NY, USA). Medium containing $30 \%$ fetal bovine serum in the lower chamber was used as chemoattractant. Suspensions were placed at $1 \times 10^{5}$ cells/well into the upper chamber in $100 \mu \mathrm{l}$ of serum-free medium and incubated in $5 \% \mathrm{CO} 2$ at $37^{\circ} \mathrm{C}$ for $48 \mathrm{~h}$. Then the filters were fixed with $4 \%$ paraformaldehyde and stained with $0.1 \%$ crystal violet, the average number of cells on the membrane of lower chamber from five randomly chosen microscope fields was counted. For wound-healing assay, the A549 cells were seeded in 12-well plates and allowed to grow until their concentration reached $90 \%$, then scratched the cell monolayer with a micropipette tip and cultured in serum-free medium. Microphotographs were taken at $48 \mathrm{~h}$. 


\section{Cell viability assay}

MTT assay was performed to measure A549 cells viability after cells were seeded into 96-well plates and incubated at $37^{\circ} \mathrm{C}$ for 2 days. A549 cells viability was calculated by the survival rate (the ratio of the OD value at $570 \mathrm{~nm}$ between the experimental group and the control group). All experiments were repeated three times independently and three samples per group.

\section{Results}

\section{Relationship between the mRNA levels of ASICs and the clipathological parameters of patients with lung cancer}

To investigate the mRNA expressions of ASICs factors between lung cancer and normal lung tissues, the GEPIA dataset (http://gepia.cancer-pku.cn/)was used. The results showed that the expression levels of ASIC1, ASIC2, ASIC3 and ASIC4 were not high in tumor and normal lung tissues, and mRNA expressions of ASIC1and ASIC3 in LUSC tissues were higher compared with normal tissues (Figure 1A, $p<0.05$ ). Next, we also analyzed the expression of ASICs with tumor stage for LUAD and LUSC. The results indicated that the expression level of ASIC2 group significantly varied, whereas ASIC1, ASIC3 and ASIC4 groups were not significantly different (Figure 1B). We performed immunohistochemical staining to test ASICs proteins expression in lung cancer tissues and the counterparts and to examine the expression of ASICs in tumor. The results showed that ASIC1 and ASIC3 proteins were more highly expressed in the lung cancer tissues than the normal lung tissues (Figure 1C), however, all the expression level of ASICs were not high.

\section{Prognostic value of the ASICs mRNA expression in lung cancer patients}

To determine the prognostic values of the mRNA expression of ASICs in lung cancer patients, KaplanMeier Plotter database (http://kmplot.com/analysis/index.php?p=service\&cancer=lung) was used to analysis the survival condition between high expression and low expression. The results showed that the increased ASIC3 and ASIC4 mRNA levels were significantly associated with progression-free survival (FP) and overall survival (OS) $(p<0.05)$, and all the ASICs mRNA levels were not associated with post progression survival (PPS) $(p>0.05)$ (Figure 2). Combined the different ASICs mRNA expression in tumor and normal tissues, these results suggested that the mRNA expression levels of ASIC 3 may be useful for prediction of lung cancer patient overall survival.

\section{Effects of hypercapnic acidosis on the expression of ASIC3 in A549 cells}

To assess the effects of the HCA microenvironment, the A549 cells were cultured in $10 \% \mathrm{CO} 2$ and $90 \%$ air. Compared with the A549 cells cultured in normal environment (5\% $\mathrm{CO} 2$ and $90 \%$ air), the expression of ASIC3 significantly increased (fluorescence intensity of ASIC3, $0.43 \pm 0.07$ vs $0.79 \pm 0.11, p<0.01$ ) (Figure 3). After ASIC3 blocked by aminloride which was added to medium at various final concentrations 100 
$\mu \mathrm{M}$ for $48 \mathrm{~h}$ in HCA microenvironment, the expression of ASIC3 significantly decreased(fluorescence intensity of ASIC3, $0.79 \pm 0.11$ vs $0.17 \pm 0.05, p<0.01$ ) (Figure 3 ).

\section{Hypercapnic acidosis microenvironment promoted EMT of A549 cells}

We have showed that the mRNA expression levels of ASIC3 may be useful for prediction of lung cancer patient overall survival, and the expression of ASIC3 was relatively higher in HCA microenvironment, therefore, we performed transwell assay and wound-healing assay of A549 cells in HCA microenvironment to further determine their roles in EMT, and also performed MTT assay to measure A549 cells viability. The results showed that the invasive ability of A549 cells was promoted in HCA microenvironment. Correspondingly, the invasive ability of A549 cells was inhibited after ASIC3 blocked (Figure 4A and 4B). Similarly, the migrate ability of A549 cells was also promoted in HCA microenvironment and suppressed after ASIC3 blocked (Figure 4C). However, inhibition of ASIC3 did not display significant effect on viability of A549 cells (Figure 4D). Collectively, these results suggested that A549 cells have the increased activity of invasion and migration in HCA microenvironment, and ASIC3 plays an important role in these processes.

\section{Discussion}

HCA which occurs due to an excess of $\mathrm{CO} 2$ in the blood has been associated with improvement in patients with ALI/ARDS, and the permissive and even therapeutic hypercapina have been proposed in treating the ALI/ARDS patients [15-17]. However, there are also studies showed that hypercapnia has some harmful effects, such as inhibiting alveolar epithelial function and epithelial cell repair, suppressing innate immunity/host defence and airway function[18]. In this study, we investigated the effects of HCA microenvironment on EMT of A549 cells. Bioinformatics data were used and foundthat the mRNA expression levels of ASIC3 may be useful for prediction of lung cancer patient overall survival. Next, we investigated the relationship between ASIC3 and the effects of HCA on EMT of the A549 lung cancer cells. The results showed that the expression of ASCl3 increased and the EMT of A549 cells promoted after exposure to a HCA (10\%CO2+90\% air) environment, and blocking ASIC3 could suppress these effects. Collectively, our results demonstrated that HCA microenvironment promoted the EMT of human lung cancer A549 cells through ASIC3, which also may affect the overall survival of lung cancer patients.

The acidic microenvironment is closely related to the proliferation $[28,29]$, invasion and metastasis of tumors [30], which is carried out mainly by inducing new angiogenesis, escaping immune surveillance, and EMT. Therefore, the targeted tumor microenvironment may become part of future anti-tumor therapy[31]. Studies showed that the tumor cells always in hypoxia environment for the tumor cells grows fast and the new blood vessel function is incomplete [32-34]. However, a large number of studies have confirmed that tumor cells, even with sufficient oxygen supply, are mainly anaerobic glycolysis to obtain energy, which is known as the "Warburg effect" [35-37]. Hypoxia is often associated with $\mathrm{CO} 2$ accumulation, so HCA microenvironment may play an important role in cancer progression and evolution. In this study, transwell assay and wound-healing assay were used to determine HCA microenvironment 
effects in EMT of lung cancer A549 cells. The results showed that HCA microenvironment could increase the activity of invasion and migration of A549 cells in vitro. But it is not clear yet how HCA microenvironment affects EMT of lung cancer A549 cells.

In this study, we used bio-information technology to investigate the mRNA expressions of ASICs factors between lung cancer and normal lung tissues, and the GEPIA dataset showed that the expression levels of ASICs were not high in tumor and normal lung tissues, and only ASIC1and ASIC3 mRNA expressions had difference, and immunohistochemical staining showed the same results as GEPIA dataset. We also investigated the prognostic values of the mRNA expression of ASICs in lung cancer patients by KaplanMeier Plotter database. It showed that the increased ASIC3 and ASIC4 mRNA levels were significantly associated with FP and OS. So the mRNA expression levels of ASIC3 may be useful for prediction of lung cancer patient overall survival. Although ASICs are expressed in most of physiological and pathological functions in neurological diseases [38, 39], some studies have shown that ASIC1 and ASIC3 are involved in acid-induced EMT to promote tumor metastasis [23]. Interestingly, HCA microenvironment could increase the expression of ASIC3 in A549 cells, and the invasion and migration of A549 cells were suppressed after blocking ASIC3 in HCA microenvironment. Therefore, our results suggest that ASIC3 which regulating HCA micoenvironment induced EMT of A549 cells, at least partially, may be contributed to the metastasis of lung cacer and the survival of lung cancer patients.

In conclusion, our study highlights a mechanism for HCA micoenvironment induce EMT in lung cancer cells through ASIC3, which may affect to the FP and OS of lung cancer patients. Therefore, ASIC3 may represent a possible target for therapeutic intervention in lung cancer. Alternatively, strengthening the intervention of HCA may be an important measure in the clinical treatment of lung cancer.

\section{Declarations}

\section{Ethics approval and consent to participate}

Written informed consent was obtained from all participants. The study complied with the Declaration of Helsinki and was approved by the Ethics Committee of the First Affiliated Hospital of Nanchang University.

\section{Consent for publication}

Not applicable.

\section{Availability of data and materials}

The datasets generated and/or analysed during the current study are available in the Gene Expression Profiling Interactive Analysis and Kaplan-Meier Plotter database repository. [http://gepia.cancer-pku.cn/ and http://kmplot.com/analysis/index.php?p=service\&cancer=lung]

\section{Competing interests}


The authors declare that they have no competing interests.

\section{Funding}

This study was supported by the Foundation of Science and Technology of Jiangxi Province Plan (NO.20202BABL206071).

\section{Authors' contributions}

This study conception and design were performed by Liangchao Qu. Dingde Long , Xingjun Fang and Peihua Yuan wrote the main manuscript text, and Hongtao Li confirm the authenticity of all the raw data. Critical revision of the manuscript was given by all authors. All authors reviewed the manuscript.

\section{Acknowledgements}

We thank Professor Lei Zhou of Virginia Commonwealth University for the language modification.

\section{References}

[1] Chen W, Zheng R, Zeng $\mathrm{H}$ and Zhang S. Epidemiology of lung cancer in C hina. Thoracic cancer 2015; 6: 209-215.

[2] Wu C, Li M, Meng H, Liu Y, Niu W, Zhou Y, Zhao R, Duan Y, Zeng Z and Li X. Analysis of status and countermeasures of cancer incidence and mortality in China. Science China Life Sciences 2019; 62: 640647.

[3] Organization WH. Global status report on alcohol and health 2018. World Health Organization, 2019.

[4] Little AG, Rusch VW, Bonner JA, Gaspar LE, Green MR, Webb WR and Stewart AK. Patterns of surgical care of lung cancer patients. The Annals of thoracic surgery 2005; 80: 2051-2056.

[5] Stewart B and Wild CP. World cancer report 2014. 2014;

[6] Friedlaender A, Nouspikel T, Christinat Y, Ho L, McKee T and Addeo A. Tissue-Plasma TMB Comparison and Plasma TMB Monitoring in Patients With Metastatic Non-small Cell Lung Cancer Receiving Immune Checkpoint Inhibitors. Frontiers in Oncology 2020; 10: 142.

[7] Lambert AW, Pattabiraman DR and Weinberg RA. Emerging biological principles of metastasis. Cell 2017; 168: 670-691.

[8] Vargas AJ and Harris CC. Biomarker development in the precision medicine era: lung cancer as a case study. Nature Reviews Cancer 2016; 16: 525.

[9] Javle M, Gibbs J, Iwata K, Pak Y, Rutledge P, Yu J, Black JD, Tan D and Khoury T. Epithelialmesenchymal transition (EMT) and activated extracellular signal-regulated kinase ( $p$-Erk) in surgically 
resected pancreatic cancer. Annals of surgical oncology 2007; 14: 3527-3533.

[10] Liu J, Li C, Zhang L, Liu K, Jiang X, Wang X, Yang L, Liang W, Liu K and Hu J. Association of tumourassociated macrophages with cancer cell EMT, invasion, and metastasis of Kazakh oesophageal squamous cell cancer. Diagnostic pathology 2019; 14: 55.

[11] Milotti E and Chignola R. Emergent properties of tumor microenvironment in a real-life model of multicell tumor spheroids. PloS one 2010; 5:

[12] Barar $\mathrm{J}$ and Omidi Y. Dysregulated $\mathrm{pH}$ in tumor microenvironment checkmates cancer therapy. Biolmpacts: BI 2013; 3: 149.

[13] Gupta S, Roy A and Dwarakanath BS. Metabolic cooperation and competition in the tumor microenvironment: implications for therapy. Frontiers in oncology 2017; 7: 68.

[14] Payen VL, Porporato PE, Baselet B and Sonveaux P. Metabolic changes associated with tumor metastasis, part 1: tumor $\mathrm{pH}$, glycolysis and the pentose phosphate pathway. Cellular and Molecular Life Sciences 2016; 73: 1333-1348.

[15] O'Croinin D, Chonghaile MN, Higgins B and Laffey JG. Bench-to-bedside review: permissive hypercapnia. Critical Care 2004; 9: 51.

[16] Rimensberger PC, Cheifetz IM and Group PALICC. Ventilatory support in children with pediatric acute respiratory distress syndrome: proceedings from the Pediatric Acute Lung Injury Consensus Conference. Pediatric Critical Care Medicine 2015; 16: S51-S60.

[17] Vadász I, Hubmayr RD, Nin N, Sporn PH and Sznajder Jl. Hypercapnia: a nonpermissive environment for the lung. American journal of respiratory cell and molecular biology 2012; 46: 417-421.

[18] Shigemura M, Lecuona E and Sznajder Jl. Effects of hypercapnia on the lung. The Journal of physiology 2017; 595: 2431-2437.

[19] Wemmie JA, Price MP and Welsh MJ. Acid-sensing ion channels: advances, questions and therapeutic opportunities. Trends in neurosciences 2006; 29: 578-586.

[20] Waldmann R, Champigny G, Bassilana F, Heurteaux C and Lazdunski M. A proton-gated cation channel involved in acid-sensing. Nature 1997; 386: 173-177.

[21] Wemmie JA, Taugher RJ and Kreple CJ. Acid-sensing ion channels in pain and disease. Nature Reviews Neuroscience 2013; 14: 461-471.

[22] Kirkegaard T and Jäättelä M. Lysosomal involvement in cell death and cancer. Biochimica et Biophysica Acta (BBA)-Molecular Cell Research 2009; 1793: 746-754. 
[23] Zhu S, Zhou H-Y, Deng S-C, Deng S-J, He C, Li X, Chen J-Y, Jin Y, Hu Z-L and Wang F. ASIC1 and ASIC3 contribute to acidity-induced EMT of pancreatic cancer through activating $\mathrm{Ca} 2+/ \mathrm{RhoA}$ pathway. Cell death \& disease 2017; 8: e2806-e2806.

[24] Shao L, Chen Y, Shaoqun $X$ and Qiu $H$. Expression of acid sensing ion channel 3 in the lung tissue of rats with, acute lung injury. Chinese Journal of Emergency Medicine 2009; 18: 466-470.

[25] Tang Z, Li C, Kang B, Gao G, Li C and Zhang Z. GEPIA: a web server for cancer and normal gene expression profiling and interactive analyses. Nucleic acids research 2017; 45: W98-W102.

[26] Győrffy B, Surowiak P, Budczies J and Lánczky A. Online survival analysis software to assess the prognostic value of biomarkers using transcriptomic data in non-small-cell lung cancer. PloS one 2013; 8:

[27] Wu S-Y, Li M-H, Ko F-C, Wu G-C, Huang K-L and Chu S-J. Protective effect of hypercapnic acidosis in ischemia-reperfusion lung injury is attributable to upregulation of heme oxygenase-1. PLoS One 2013; 8:

[28] Tian X-P, Wang C-Y, Jin X-H, Li M, Wang F-W, Huang W-J, Yun J-P, Xu R-H, Cai Q-Q and Xie D. Acidic microenvironment up-regulates exosomal miR-21 and miR-10b in early-stage hepatocellular carcinoma to promote cancer cell proliferation and metastasis. Theranostics 2019; 9: 1965.

[29] Xing Y, Zhao S, Zhou BP and Mi J. Metabolic reprogramming of the tumour microenvironment. The FEBS journal 2015; 282: 3892-3898.

[30] Estrella V, Chen T, Lloyd M, Wojtkowiak J, Cornnell HH, Ibrahim-Hashim A, Bailey K, Balagurunathan Y, Rothberg JM and Sloane BF. Acidity generated by the tumor microenvironment drives local invasion. Cancer research 2013; 73: 1524-1535.

[31] Kanapathipillai M, Brock A and Ingber DE. Nanoparticle targeting of anti-cancer drugs that alter intracellular signaling or influence the tumor microenvironment. Advanced drug delivery reviews 2014; 79 : 107-118.

[32] Zhang S, Li M, Zhang D, Xu S, Wang X, Liu Z, Zhao X and Sun B. Hypoxia influences linearly patterned programmed cell necrosis and tumor blood supply patterns formation in melanoma. Laboratory investigation 2009; 89: 575-586.

[33] Lin Q and Yun Z. Impact of the hypoxic tumor microenvironment on the regulation of cancer stem cell characteristics. Cancer biology \& therapy 2010; 9: 949-956.

[34] Paardekooper LM, Vos W and van den Bogaart G. Oxygen in the tumor microenvironment: effects on dendritic cell function. Oncotarget 2019; 10: 883.

[35] Liberti MV and Locasale JW. The Warburg effect: how does it benefit cancer cells? Trends in biochemical sciences 2016; 41: 211-218. 
[36] Pavlides S, Whitaker-Menezes D, Castello-Cros R, Flomenberg N, Witkiewicz AK, Frank PG, Casimiro MC, Wang C, Fortina P and Addya S. The reverse Warburg effect: aerobic glycolysis in cancer associated fibroblasts and the tumor stroma. Cell cycle 2009; 8: 3984-4001.

[37] Pereira-Nunes A, Afonso J, Granja S and Baltazar F. Lactate and lactate transporters as key players in the maintenance of the warburg effect. In: editors. Tumor Microenvironment. Springer; 2020. p. 51-74.

[38] Chu X-P and Xiong Z-G. Physiological and pathological functions of acid-sensing ion channels in the central nervous system. Current drug targets 2012; 13: 263-271.

[39] Kweon H-J and Suh B-C. Acid-sensing ion channels (ASICs): therapeutic targets for neurological diseases and their regulation. BMB reports 2013; 46: 295.

\section{Figures}
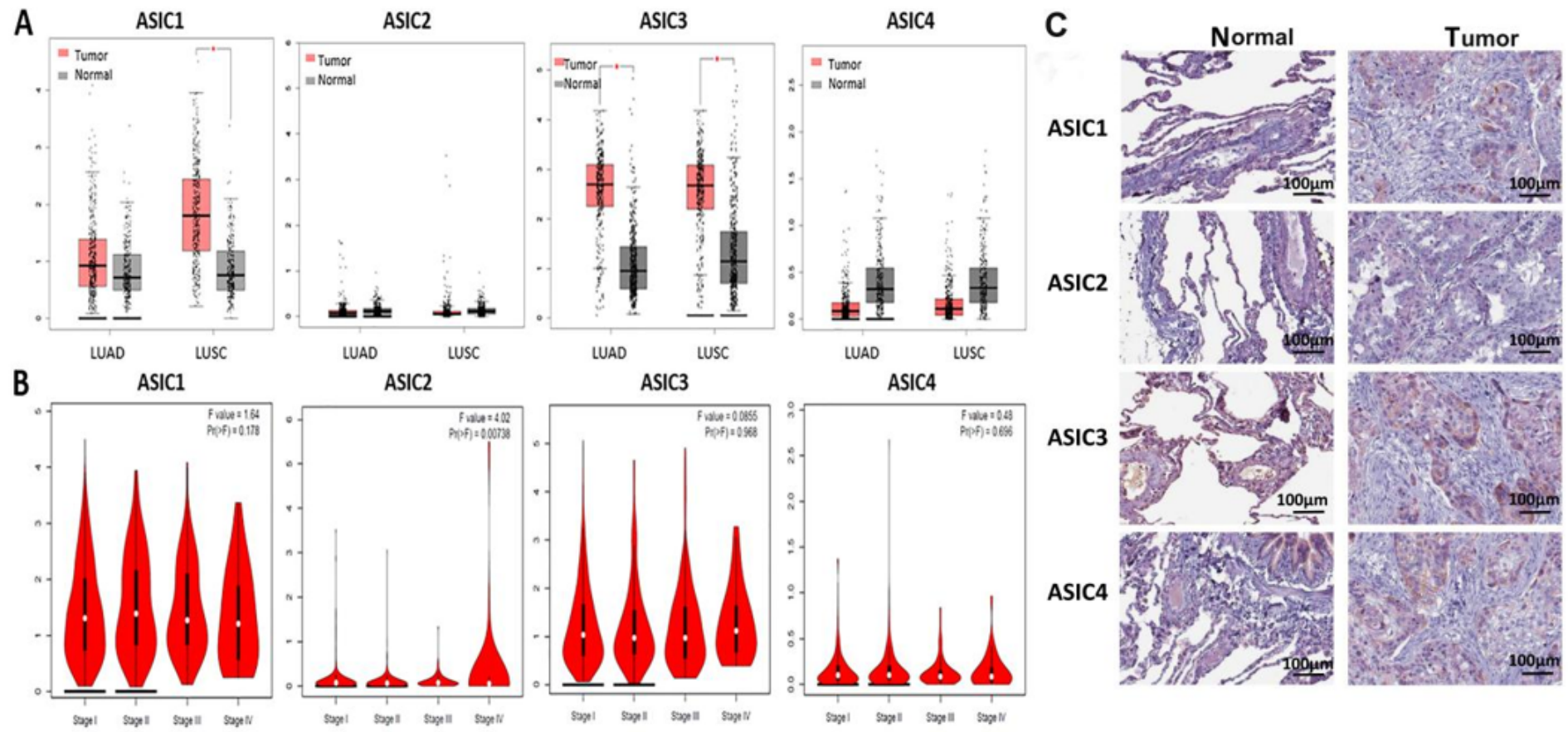

\section{Figure 1}

The expression of ASICs in lung cancer. A. The expression of ASICs mRNA levels in LUAD and LUSC (GEPIA). B. Correlation between ASICs expression and tumor stage in lung cancer patients (GEPIA). C. The expression of ASICs in lung cancer tissue by Immunohisto-chemical staining. 

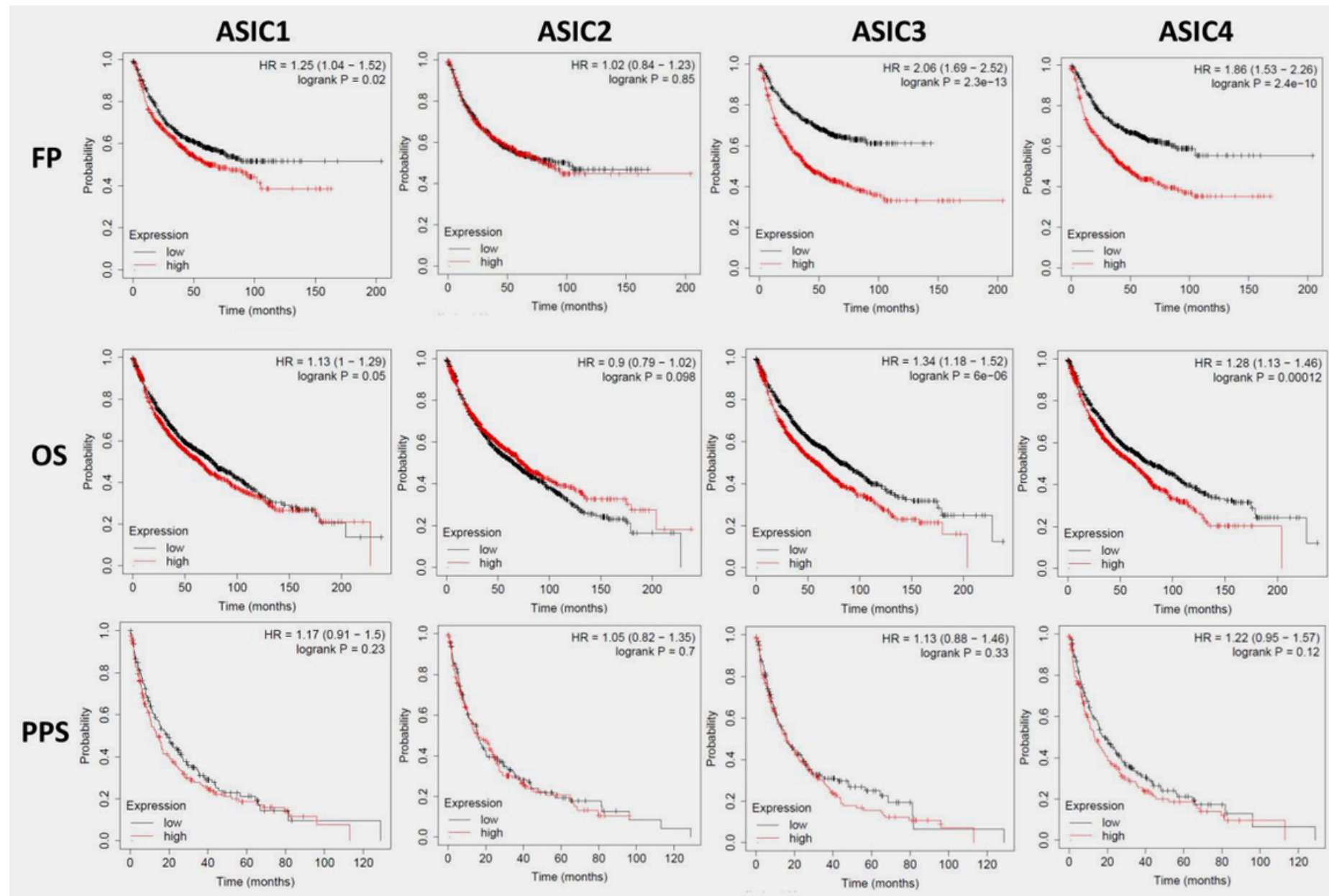

\section{Figure 2}

The prognostic value of mRNA level of ASICs factors in lung cancer patients (Kaplan-Meier plotter). 


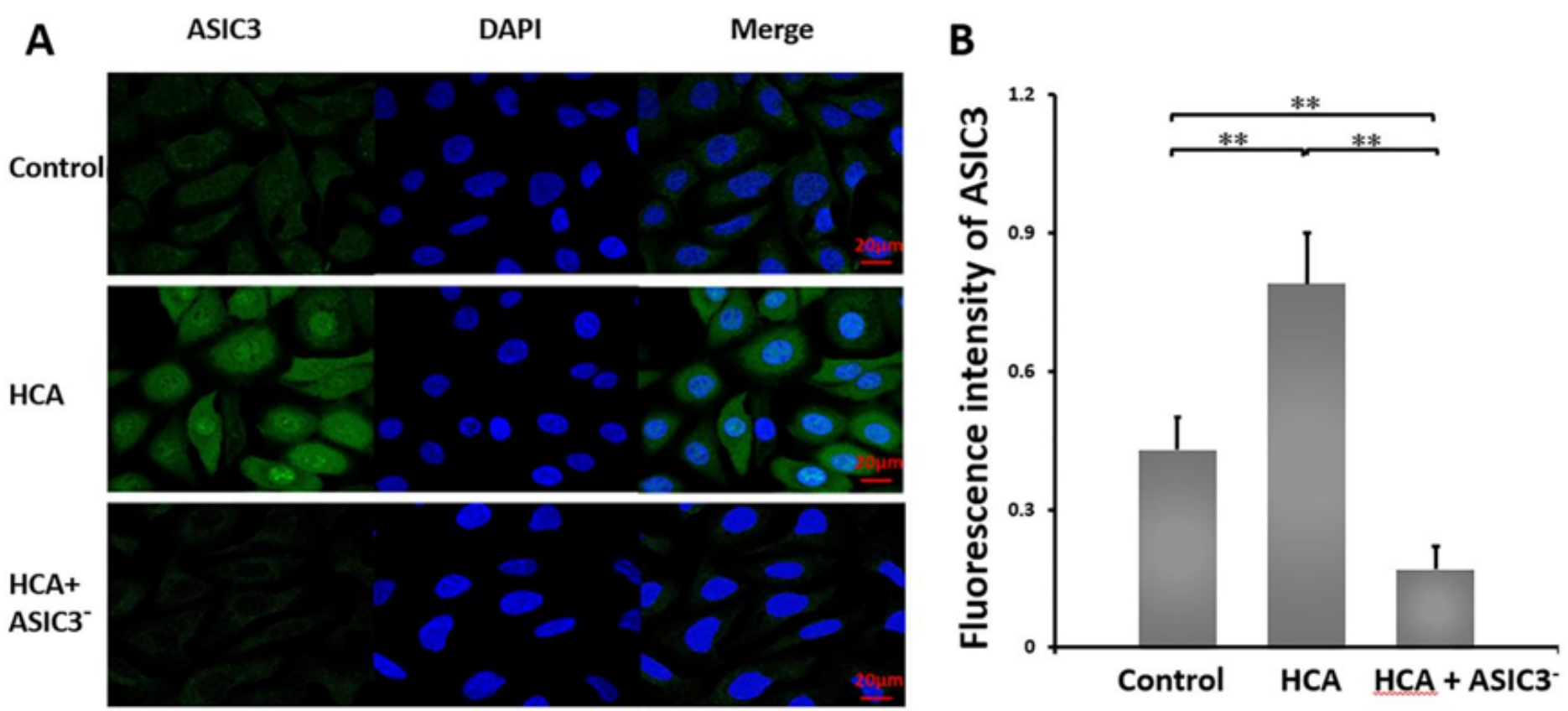

Figure 3

Effects of hypercapnic acidosis on the expression of ASIC3 in A549 cells. A. Representative images of ASIC3 expression in different groups of A549 cells (immunofluorescence staining). The green fluorescence shows ASIC3, the blue fluorescence shows the nucleus, and the combined pink shows the expression of ASIC3 in A549 cells. B. ASIC3 fluorescence intensity results were showed in each group. The data are shown as the mean \pm s.e.m. ${ }^{\star \star} p<0.01$. 
A

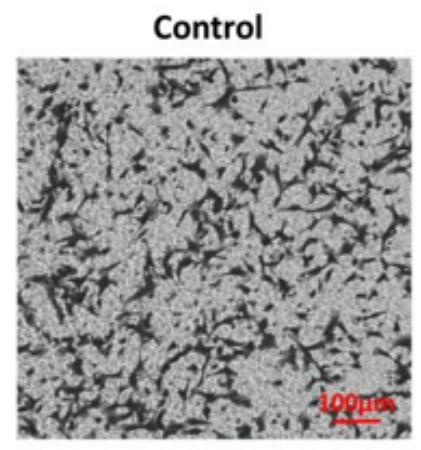

C

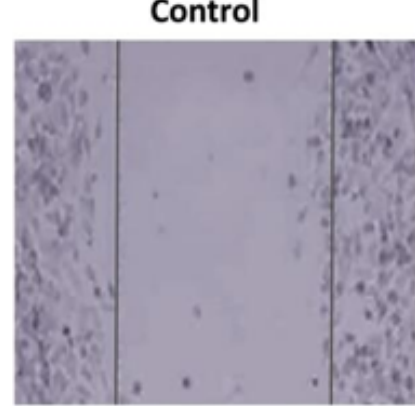

HCA

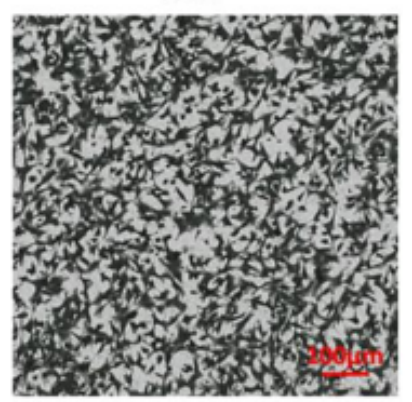

HCA

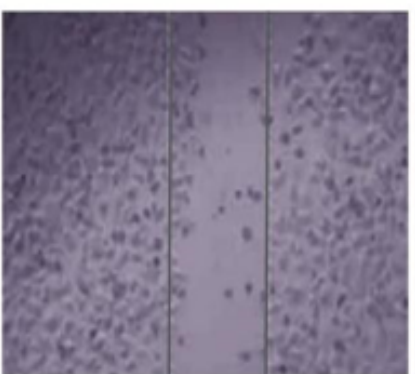

HCA + ASIC3-

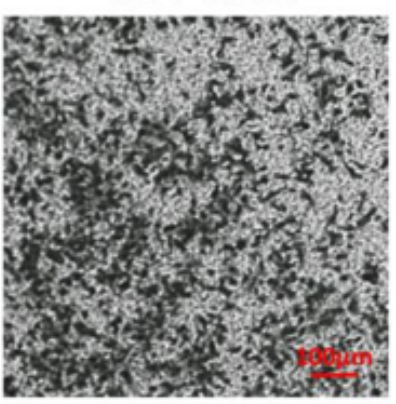

$\mathrm{HCA}_{+} \mathrm{ASIC3}^{-}$

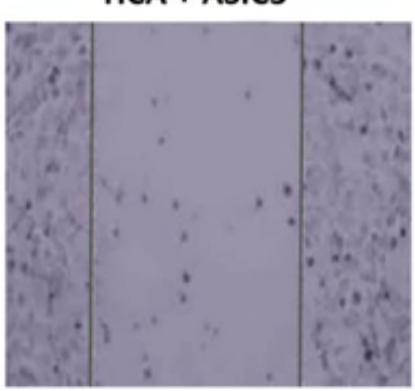

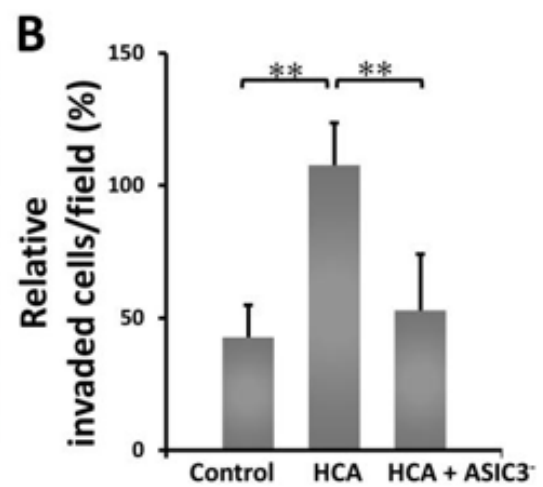

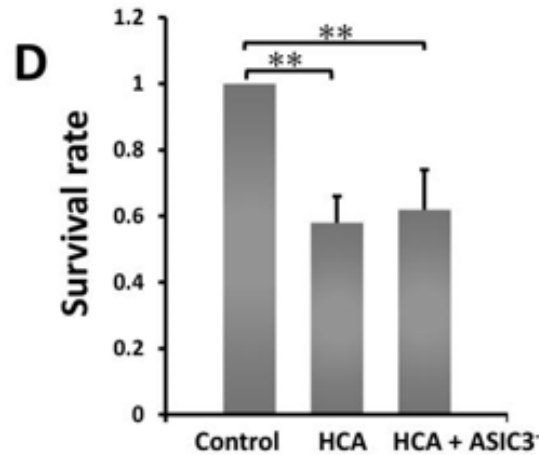

\section{Figure 4}

Hypercapnic acidosis microenvironment promoted EMT of A549 cells. A. The invasive ability was evaluated in different groups of A549 cells by Transwell assay. B. The percentage of invasion cells per field. C. The migration ability was measured in different groups of A549 cells by wound-healing assay. D. The cell viability was measured in different groups of A549 cells by MTT assay. The data are shown as the mean \pm s.e.m. ${ }^{\star \star} p<0.01$. 